CONSTRUCTION DE LA LGV SEA TOURS-BORDEAUX ET POLITIQUE DE RECRUTEMENT LOCAL : ANALYSE DE LA COORDINATION DES ACTEURS PUBLICS ET PRIVES

HSL TOURS - BORDEAUX CONSTRUCTION AND EMPLOYMENT POLICY: ANALYSIS OF THE COORDINATION BETWEEN PUBLIC AND PRIVATE STAKEHOLDERS

A paraitre sous cette forme dans le prochain numéro varia de la revue

\title{
AUTEURS
}

\section{Etienne FOUQUERAY}

CRIEF EA2249 - Université de Poitiers, LISEA

etienne.fouqueray@univ-poitiers.fr

Elodie MANCEAU-ANTONIAZZI ${ }^{1}$

UMR 8504 Géographie-cités

manceau.elodie@gmail.com

Mots clés: Construction, LGV SEA, emploi, formation, système d'acteurs, gouvernance, proximité.

Keywords: Construction, HSL Tours-Bordeaux, employment, Stakeholders, governance, proximity.

Les propos et les opinions exprimés dans cet article n'engagent que les auteurs et n'engagent pas les sociétés, auxquelles ils contribuent ou dont ils peuvent être associés ou employés.

Résumé

\footnotetext{
${ }^{1}$ Elodie Manceau-Antoniazzi a été responsable de l'observatoire socio-économique de la LGV SEA Tours-Bordeaux entre 2012 et 2015. Depuis, elle a rejoint les Services de l'État comme chargée des relations institutionnelles avec le territoire pour le Lyon-Turin ferroviaire (section internationale) et participe dans ce cadre à la démarche "Grand chantier" prévue par le CIADT de décembre 2003.
} 
Lancée en 2011, la construction de la Ligne à Grande Vitesse Sud Europe Atlantique (LGV SEA) entre Tours et Bordeaux a mobilisé près de 9000 salariés au plus fort des travaux à l'été 2013. La mobilisation massive de ces ressources humaines a nécessité la mise en œuvre d'une politique d'emploi et de formation adaptée de la part du constructeur et des parties prenantes locales au projet. En particulier, le recrutement d'environ 2000 salariés locaux par le constructeur a été permis par l'instauration de relations partenariales entre acteurs locaux et nationaux de l'emploi et de la formation. Après avoir souligné le manque d'évaluation des dispositifs «emploiformation » accompagnant les grands chantiers d'infrastructure de transport, cet article interroge la construction du dispositif «emploi-formation » et apporte des premiers éléments d'évaluation du système de gouvernance local ayant permis le recrutement de ces personnels.

\begin{abstract}
The construction of the High Speed Line South Europe Atlantic (LGV SEA) between Tours and Bordeaux started in 2011 and required about 9000 employees at the peak in summer 2013. To reach this goal the construction company and local stakeholders had to develop an employment and training policy adapted to the project. In particular, the recruitment of about 2000 local employees by the construction company has been possible through a partnership between both local and national employment and training stakeholders. Because of the lack of assessment of "employment-training" policies accompanying major transport infrastructure projects, this paper aims to analyze the plan for "employment training" implemented on this project in order to assess the effectiveness of local governance system that allowed the recruitment of these employees.
\end{abstract}




\section{Introduction}

Première Ligne à Grande Vitesse française (LGV) construite et exploitée sous la forme d'un partenariat public-privé, la LGV Sud Europe Atlantique (SEA) entre Tours et Bordeaux a pour objectif de rapprocher Bordeaux et Paris d'une heure et de libérer des sillons pour le fret sur la ligne existante. Débutée en 2011, la construction de la LGV SEA, dont le financement s'élève à 7,8 milliards d'euros, doit s'achever en 2016 pour une mise en service mi-2017. L'ampleur du projet a nécessité la mise en œuvre d'une politique d'emploi et de formation adaptée dans l'ensemble des territoires traversés par la ligne afin de fournir une main d'œuvre locale qualifiée au constructeur puis d'assurer la sécurisation des parcours professionnels des personnes recrutées et formées (Convention partenariale État, Pôle Emploi, COSEA, Conseil Régional de PoitouCharentes, 2011).

Ce défi a été de taille puisque la LGV SEA longue de $340 \mathrm{~km}$ (raccordements compris) traverse trois régions (Centre, Poitou-Charentes et Aquitaine), six départements (Indre-et-Loire, Vienne, Deux-Sèvres, Charente, Charente-Maritime et Gironde), trente-cinq communautés de communes et d'agglomérations et cent treize communes (Fig. 1). Dans ce contexte géographique et économique, la politique «emploi-formation » mise en œuvre dans la construction de la LGV SEA représente un laboratoire de choix pour évaluer les relations qui existent entre acteurs sur les marchés locaux du travail dans le cadre de grands chantiers d'infrastructure de transport. En effet, contrairement aux grands chantiers de centrale nucléaire, de terminaux méthaniers ou encore d'équipements culturels, ces derniers présentent la caractéristique de s'inscrire sur un linéaire considérable impliquant plusieurs territoires, visant une population plus éclatée géographiquement et multipliant le nombre d'acteurs mobilisés autour des questions d'emploi et de formation.

Dans cet article, nous proposons à la fois d'analyser la manière dont se sont coordonnés les différents acteurs locaux, régionaux et nationaux, privés et publics, pour accompagner cette variation de main d'œuvre sur le chantier, en nous focalisant sur le recrutement de la main d'œuvre locale et l'efficacité de cette coordination à travers les témoignages des protagonistes. Nous focalisons l'analyse sur la coordination «emploi-formation » mis en place en PoitouCharentes (Figure.1, p.4) qui est la région la plus traversée par le linéaire et où les ressources humaines ont donc été les plus mobilisées.

Après avoir souligné l'intérêt de mobiliser la littérature de l'école de la proximité pour comprendre l'élaboration des politiques locales d'emploi et de formation, nous consacrons la première partie de cet article à un retour d'expériences des politiques d'emploi et de formation accompagnant les grands chantiers d'infrastructures de transport. Montrant l'absence récurrente d'évaluation de ces politiques, nous proposons une méthodologie pour analyser la coordination des parties prenantes de la politique «emploi-formation» conduite dans le cadre de la construction de la LGV SEA Tours-Bordeaux. La deuxième partie de l'article s'attache à 
décrire le contexte et les enjeux liés à l'emploi du chantier de la LGV SEA, les acteurs impliqués pour enfin interroger en troisième partie les fondements de la gouvernance locale «emploiformation » mise en œuvre autour du projet de la LGV SEA.

Fig. 1 : Le tracé de la LGV SEA et ses raccordements à la ligne classique

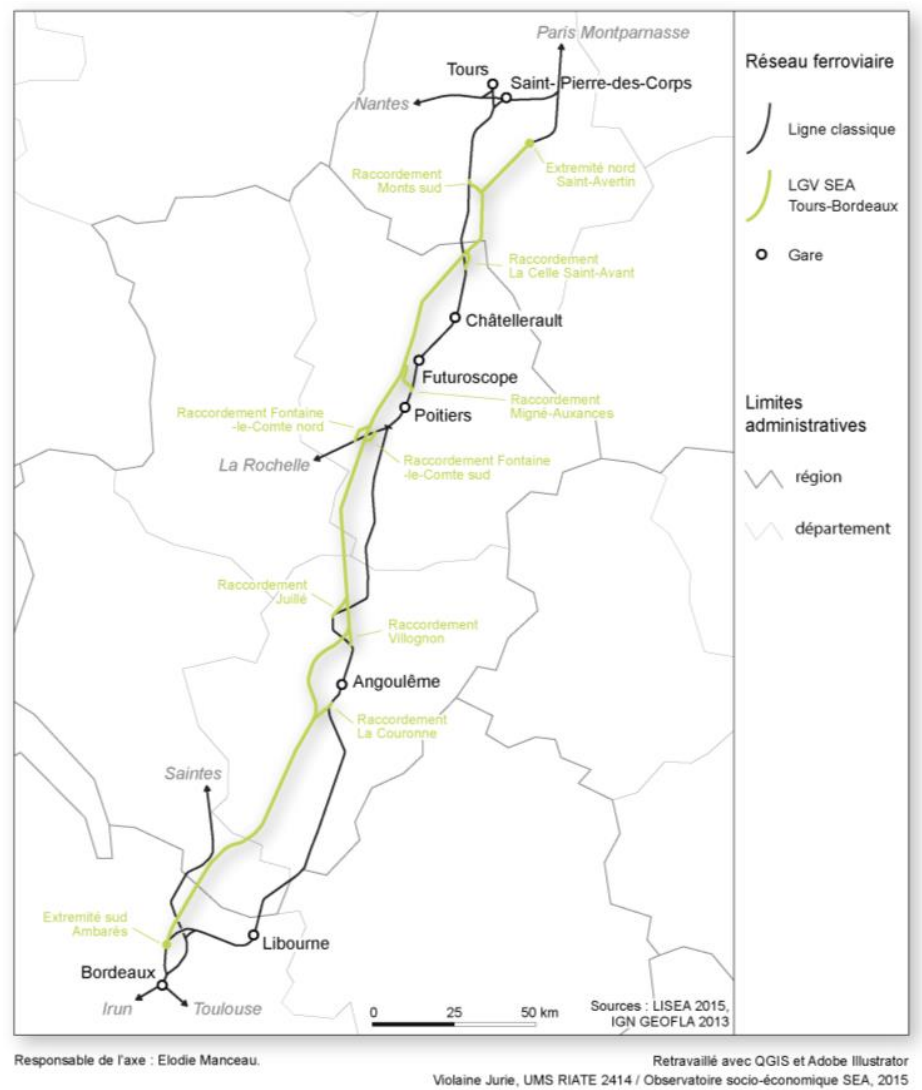

1. L'emploi et la formation sur les grands chantiers d'infrastructures de transport : revue de littérature et méthodologie

\subsection{Une analyse proximiste de la gouvernance des politiques locales « emploi-formation»}

La conception et la mise en œuvre d'une politique d'emploi et de formation dans le cadre d'un grand chantier de construction ferroviaire ou routière est l'objet d'un jeu de coordinations multiples entre acteurs locaux et nationaux, motivés par des intérêts divergents. En mobilisant la littérature de l'école de proximité sur les rapports «entreprise-territoire », nous proposons un cadre d'analyse des systèmes de gouvernance de ces politiques d'accompagnement. Celle-ci nous enseigne que les coordinations territoriales entre acteurs se fondent sur le partage de règles du jeu, le développement de relations interpersonnelles et inter-organisationnelles à l'origine du fondement d'une relation de confiance et sur le déploiement d'une organisation commune. A chacune de ces étapes, la proximité géographique entre les acteurs est essentielle pour faciliter les interactions de face à face. 
Selon ce cadre théorique, la gouvernance locale est un «processus de construction d'une proximité institutionnelle nécessaire à la réalisation d'une proximité organisationnelle entre des acteurs géographiquement proches »(Gilly, Leroux, \& Wallet, 2004). Pour Pecqueur et Zimmermann (2004, p.25), la proximité est la «capacité d'agents qui la partagent à se coordonner». Les analyses en termes de proximité différencient la proximité spatiale de la proximité non spatiale. «La proximité proprement géographique peut alors jouer un rôle de facilitateur par les externalités qu'elles produisent et aussi de constitution dynamique d'espace économique » (Pecqueur \& Zimmermann, 2004, p.33). Nous appuyant sur la décomposition retenue par Gilly et Torre (2000), Gilly et Perrat (2003) et Pecqueur et Zimmermann (2004), nous distinguons deux formes de proximités non spatiales : «organisationnelle » (interaction directe) et «institutionnelle» (interaction indirecte). Il existe une proximité institutionnelle lorsque les institutions «assurent auprès des agents concernés la diffusion d'un signal dont tout le monde dispose également (connaissance commune) et sur lequel peuvent se construire les anticipations, sans nécessiter d'interaction directe» (Ibid., p.26). Dans le cadre d'une proximité organisationnelle «la coordination est (...) obtenue soit par l'intégration au sein d'une organisation dans laquelle sont définies les pratiques individuelles et les relations entre les acteurs (...), soit par le fait d'un processus itératif sur la base de jeux répétés » (Pecqueur \& Zimmermann, 2004, p.28). La proximité institutionnelle et plus encore la proximité organisationnelle, afin d'être sources de coordinations nécessitent dès lors l'établissement d'une relation de confiance entre les acteurs (Dupuy \& Torre, 2004).

Fig. 2 : Processus de gouvernance locale

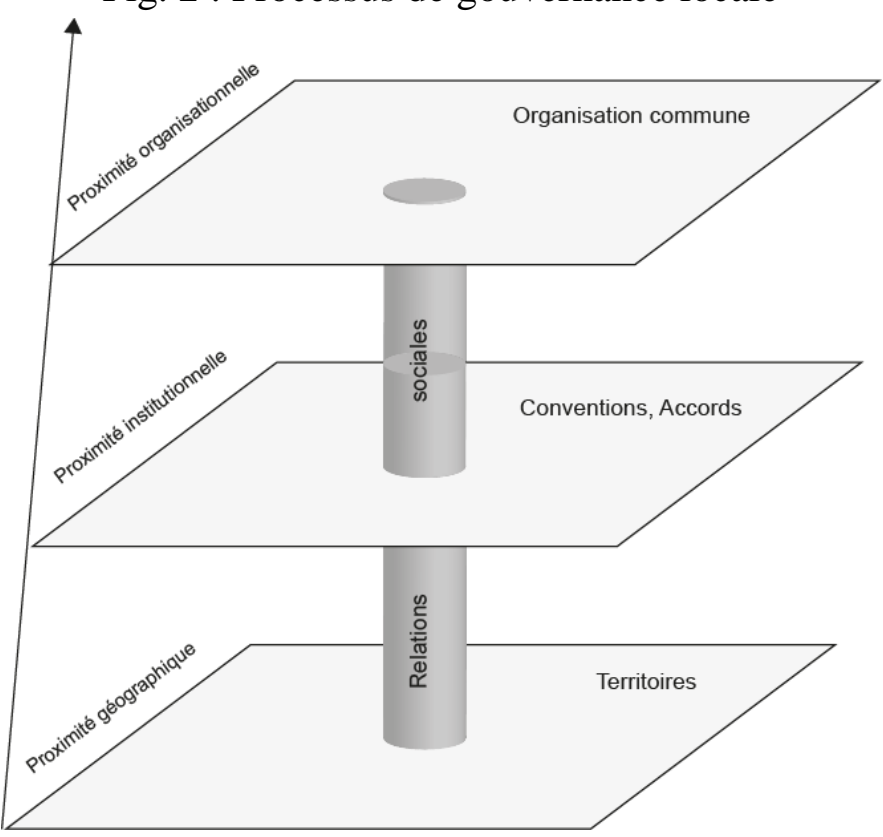

Lecture : La flèche en ordonnée indique l'ordre chronologique suivant lequel les proximités se conjuguent dans le cadre d'un processus de gouvernance locale. Pour autant il n'existe pas de hiérarchie entre ces différentes formes de proximité : l'une n'étant pas plus importante que les autres. 


\subsection{Les enjeux du recours aux ressources humaines locales lors de grands chantiers}

L'effet sur l'emploi local de la construction des grandes infrastructures de transport représente un enjeu fort pour les territoires traversés. Vus comme une opportunité de dynamisation économique et de montée en compétences des actifs locaux, les grands chantiers retiennent l'attention des élus locaux, notamment ceux dont la collectivité assume des compétences en matière d'emploi et de formation et ceux qui sont directement impactés par les travaux de génie civil. Et bien que les recherches académiques mettent en évidence une absence d'inversion de la dynamique structurelle du chômage local ${ }^{2}$ (Burmeister, 1997; Petitjean, 2003), les chiffres clés de quelques grands chantiers d'infrastructures de transport passés ou en cours, par leur ampleur, permettent de mieux comprendre le poids de ces attentes.

Par exemple, le chantier du tunnel ferroviaire sous la Manche reliant le Royaume-Uni et la France $^{3}$ avait mobilisé au plus fort du chantier 5600 personnes (Quintin, 2007). Selon le rapport du coordinateur Grand Chantier, 95\% du personnel ouvrier et $68 \%$ du personnel d'encadrement ont été recrutés dans la région (Hasiak, Jouve, \& Boijard-Lafont, 2007). Autre exemple, la construction de la LGV Rhin-Rhône avait mobilisé 2300 emplois directs entre 2006 et 2010, dont $34 \%$ de personnels locaux. Plus récemment, le chantier de la LGV Bretagne Pays de la Loire qui doit relier Le Mans à Rennes et dont la mise en service est prévue mi-2017 a mobilisé plus de 4000 salariés au plus fort du chantier dont 900 salariés embauchés localement (Eiffage, observatoire de la LGV Bretagne Pays de la Loire, 2015).

Même si les effets sur l'emploi local ne sont que temporaires et mesurés, la maximisation de ces retombées positives de court terme nécessite de pouvoir répondre à deux problématiques successives : une montée en charge rapide des effectifs et un décrutement tout aussi fulgurant, le tout pour un volume annuel d'emplois considérable (OCDE, 2002). Se pose donc, à chaque grand projet de construction d'infrastructure de transport, la question de l'appariement entre l'offre et la demande sur ce marché du travail particulier (Berion, 2002).

\footnotetext{
${ }^{2}$ Dans le cadre de la construction du tunnel sous la Manche, Burmeister (1997, p.162) constate que « le taux de chômage de la zone a suivi l'évolution structurelle de la France entière, mais avec une décrue du chômage nettement accentuée durant la période du chantier ». Parallèlement, les effectifs salariés de la zone ont crû beaucoup plus qu'ailleurs (région et France) pendant la construction et ont décru également beaucoup plus à la fin du chantier. La fin de la construction du tunnel sous la manche s'est accompagnée d'une perte de $44 \%$ des effectifs du secteur de la construction dans le Calaisis. La construction du tunnel sous la Manche a donc davantage contribué à maintenir des emplois qu'à favoriser des recrutements pérennes pour les entreprises locales sous-traitantes et fournisseuses. Burmeister explique ce résultat par i) les difficultés économiques de l'époque dans le secteur des travaux publics, ii) la taille modeste des marchés ayant eu pour conséquence une intensification du travail pour la main d'œuvre en poste, iii) l'anticipation du caractère ponctuel du chantier par les entreprises locales. A partir de données de l'Insee, Petitjean n'observe pas d'effets structurants sur les secteurs des travaux publics locaux qui seraient liés aux chantiers d'infrastructures de transport. Que ce soit pour l'A39 ou l'A77, elle ne constate pas de recrutements spécifiques au chantier pour les entreprises locales car, comme pour la construction du tunnel sous la Manche, les marchés sont de trop petite taille. Finalement, ces analyses mettent en exergue la nature éphémère des effets sur l'emploi des constructions de grandes infrastructures de transport.

${ }^{3}$ Le début des travaux a commencé le 15 décembre 1987 pour s'achever le 10 décembre 1993.
} 
Historiquement, cet appariement était garanti par un fort marché interne de l'emploi caractérisé par l'incitation à la mobilité géographique des salariés. En complément et à des fins d'ajustement, une main d'œuvre locale était recrutée dans les territoires où avaient lieu les constructions, notamment sous la forme de contrats courts (contrat à durée de chantier, CDD, intérim). Face aux problèmes sociaux et de gestion de l'emploi (turnover, conflits sociaux, etc.) ayant accompagné les projets de construction de centrales nucléaires (Flamanville, ...), les pouvoirs publics ont développé dans les années 1980 une procédure «Grand chantier», accordant une place plus importante aux marchés secondaires et locaux du travail. En effet, la démarche «Grand chantier » s'articule autour de plusieurs actions : préparer l'arrivée des chantiers sur les territoires directement concernés, accompagner leur déroulement, valoriser les opportunités offertes à cette occasion pour le développement des territoires. A plus long terme, il s'agit enfin de préparer l'après chantier. Depuis 2003, la construction de la future liaison ferroviaire Lyon-Turin est concernée par cette procédure ${ }^{4}$.

Parallèlement, la contribution financière des collectivités locales aux constructions des grandes infrastructures de transport s'est accrue sous l'effet conjoint de l'endettement croissant de l'État et des lois de décentralisation. En conséquence de cette émergence des intérêts locaux lors de grands projets de construction, les constructeurs doivent davantage arbitrer aujourd'hui qu'hier entre recrutements de personnels locaux et recrutements de personnels déplacés. Ce choix s'effectue dans un contexte de prégnance de la logique financière, caractérisée par une recherche de minimisation des coûts fixes (masse salariale), des coûts de transaction (sourcing notamment) et des coûts de formation, sous différentes contraintes (engagements politiques, compétences spécifiques, etc.).

On peut supposer que les groupes de travaux publics, en charge de ces grands projets de construction, ont intérêt économiquement, socialement et politiquement, dans la mesure où les compétences sont accessibles localement en quantité et en qualité, à se tourner vers les marchés locaux du travail pour constituer leurs ressources humaines (Hasiak et al., 2007). Le marché local de l'emploi représente une opportunité pour les postes les moins qualifiés nécessitant un effort de formation raisonnable pour les entreprises et les pouvoirs publics. Pour les emplois à fort niveau de qualification, le marché du travail s'étend à un niveau national voire international et les

\footnotetext{
${ }^{4}$ Le 18 décembre 2003, le Comité interministériel de l'aménagement et du développement du territoire (CIADT) réuni par le Premier ministre a rappelé son attachement au lancement d'une politique ambitieuse des transports «permettant la bonne insertion de nos régions dans l'espace européen et dans l'économie mondiale ». Parmi les différents projets, le Lyon-Turin ferroviaire est qualifié de «projet ambitieux destiné aux trafics voyageurs et de fret [...] qui fera l'objet vu son ampleur d'une procédure grand chantier » (Communiqué de presse). Cette procédure "Grand chantier" renvoie non seulement au nombre de salariés prévus sur le chantier (2 800 salariés au plus fort du chantier) mais aussi au territoire atypique dans lequel s'inscrit la future liaison ferroviaire et au caractère hautement technique du chantier qui se réalise en souterrain.
} 
compétences nécessaires demandent de nombreuses années de formation, ne permettant pas la mise en œuvre d'une politique locale de recrutements.

Dans ce contexte, les acteurs institutionnels du marché local du travail représentent des partenaires privilégiés pour le constructeur car sans arrangements institutionnels, les frictions sont telles sur le marché du travail des constructions de grandes infrastructures de transport qu'il est difficile d'envisager l'appariement entre une main d'œuvre locale et un constructeur multinational (Mortensen \& Pissarides, 1994). La première est, en effet, essentiellement non qualifiée et, pour partie, en situation d'exclusion sociale tandis que le second a une connaissance imparfaite de ces marchés locaux du fait de sa présence éphémère sur les territoires (Raveyre, 2005a).

\subsection{Les politiques locales "emploi-formation" sur les grands chantiers d'infrastructures de transport : une absence d'évaluation}

Afin de répondre à l'objectif de recrutements locaux, les parties prenantes publiques et privées en France comme en Europe ont élaboré sur différents projets de construction d'infrastructure de transport emblématiques - à l'image du Tunnel sous la Manche ou encore de la LGV Rhin-Rhône - de véritables gouvernances territoriales ${ }^{5}$. La gouvernance territoriale est définie par Dupuy, Leroux et Wallet (2003) comme « la construction de nouvelles formes de territorialités fondées sur des mécanismes complexes de codécisions, et sur la capacité collective d'innovation et de mobilisation des compétences et des savoirs complémentaires. Le territoire devient un espace de projet, un espace de création collective de ressources, construit sur la base de stratégies de coopération qui allient des acteurs publics (collectivités territoriales, etc.), privés, mais aussi sociaux (associations, etc.) » (Dupuy, Leroux, Wallet, 2003, Manceau, 2011).

Ces exemples ne sont pas exhaustifs mais ils ont l'avantage de témoigner des différents types de dispositifs «emploi-formation» aboutissant à la mise en place d'une gouvernance locale accompagnant la construction de grandes infrastructures de transport. Paradoxalement, tous ces systèmes de gouvernance, le plus souvent à géométrie variable, présentent la particularité de ne pas avoir été évalués au regard des objectifs qui leur avaient été assignés. Le périmètre de ces derniers peut diverger d'un projet de construction à un autre. Cependant, figure à chaque fois comme objectif de la gouvernance locale le recrutement de personnels locaux pour répondre aux besoins de main d'œuvre du constructeur. Au-delà de cet objectif central, un second est apparu de manière de plus en plus prégnante au fil des années : la sécurisation professionnelle post-chantier des personnes ayant bénéficié de la politique mise en œuvre. En revanche, l'efficacité de la politique locale «emploi/formation » ne peut être évaluée à l'aune de la réalisation de l'infrastructure dans les délais impartis puisque le recours au tissu local est seulement une option

${ }^{5}$ Cette gouvernance est par exemple en cours de construction dans le cadre du projet Lyon-Turin ferroviaire, dont l'investissement est de plus de 8 milliards d'euros, et s'inspire étroitement de la gouvernance mise en place dans le cadre du projet de la LGV SEA Tours-Bordeaux. 
parmi d'autres d'accès aux ressources humaines. En cela, il faut différencier les objectifs du maître d'ouvrage des objectifs de la politique « emploi/formation ».

Le système de gouvernance «emploi-formation » mis en place dans le cadre du Tunnel sous la Manche se caractérise par l'imbrication de plusieurs instances de concertation, de coordination et de pilotage impulsées par le Conseil Régional du Nord-Pas-de-Calais d'une part et l'État d'autre part (Hasiak et al., 2007; Quintin, 2007) qui aboutit à la signature d'un protocole en 1986. L'État n'a pas adopté la procédure «Grand chantier » mais l'esprit de celle-ci a guidé la gouvernance mise en œuvre (Quintin, 2007) avec comme objectifs de « favoriser l'accueil, le recrutement et la formation de main d'œuvre, et de profiter de la dimension du chantier pour développer les ressources économiques et humaines de la région » (Hasiak et al., 2007, p.11).

Trois décisions gouvernementales ont orienté les modalités de la coordination entre les parties prenantes :

(i) Tout d'abord, un coordonnateur a été nommé par arrêté du préfet de Région.

(ii) Ensuite, un groupe administratif permanent (GAP) a été institué avec comme responsable de l'animation le SGAR (Secrétariat Général aux Affaires Régionales) et comme secrétaire le coordonnateur. Ce GAP « regroupe autour des maîtres d'ouvrage, les services de l'État concernés par l'emploi et la formation (DDTEFP) ainsi que les organismes publics intervenant dans ces domaines (ANPE, CCI, UPI) » (Quintin, 2007).

(iii) Enfin, un comité de pilotage éducatif a été créé avec pour mission de fournir une main d'œuvre locale qualifiée en adéquation avec les besoins de recrutement des entreprises (Quintin, 2007).

Afin d'exercer un pouvoir de négociation dans la gouvernance mise en œuvre, le Conseil Régional a mis en place parallèlement un groupe de concertation avec le concessionnaire sur les thématiques de l'emploi et de la formation. L'animation de ce groupe était directement assurée par des élus, symbolisant la forte implication du Conseil Régional (Hasiak, Jouve, \& BoijardLafont, 2007). Pour rendre opérationnel le dispositif institutionnel mis en œuvre, une équipe de l'ANPE dédiée à la construction du tunnel s'est installée dans des bâtiments proches de la construction de janvier 1987 à 1994. En plus de ces instances, les acteurs publics nationaux et locaux et le concessionnaire se sont associés dans la création d'un dispositif supplémentaire, dénommé «BATIRE», afin de favoriser l'identification des besoins en main d'œuvre, en complément des dispositifs déjà en place, notamment celui de l'ANPE.

Finalement cet enchevêtrement de dispositifs a abouti sur la période 1987-1994 au financement de 900000 heures de formation pour 2340 stagiaires et un montant de plus de 8 millions d'euros, dont 6 millions d'euros par les pouvoirs publics (État, Conseil Régional, ANPE) et 2 millions d'euros par les entreprises participant à la construction (Quintin, 2007). La gouvernance mise en œuvre dans le cadre de la construction du tunnel sous la Manche s'est inscrite dans une double 
démarche de suivi et de concertation impulsée par le Conseil Régional pour impliquer l'État dans la gestion du chantier tout en gardant une action décentralisée à travers la formation professionnelle (Hasiak et al., 2007). La politique «emploi-formation » sur ce projet a été guidée par une gouvernance à deux têtes, locale et nationale, symbolisant la volonté des acteurs locaux de se saisir de l'opportunité de la construction comme vecteur de développement économique pour le territoire. Il est possible de faire l'hypothèse que cette organisation a été source de conflits mais aussi de contre-pouvoirs nécessaires à la négociation et à l'action collective. Toutefois, l'absence d'évaluation du processus à l'œuvre et de son efficacité ne permet pas d'en tirer des enseignements objectifs.

A l'inverse du tunnel sous la Manche, la construction de la Branche EST de la LGV Rhin-Rhône (2006-2011) s'est caractérisée par la mise en œuvre d'une instance unique de coordination, de pilotage et d'exécution de la politique «emploi-formation» associée au projet (Réseau Ferré de France, 2010). Sur décision du préfet, Pôle Emploi a assuré le leadership de la gouvernance d'une cellule emploi LGV dont l'objectif était de répondre aux besoins de main d'œuvre des entreprises en mobilisant des actifs locaux, dont une part était en situation d'insertion. Une vingtaine de partenaires ont été associés à cette démarche, parmi lesquels sept ont joué un rôle particulièrement moteur : Pôle Emploi, l'AFPA ${ }^{6}$, la Direccte Franche-Comté, le Conseil Général de Haute-Saône, la MIFE 907, le PLIE ${ }^{8}$ du Pays de Montbéliard et le PLIE du Grand Besançon. Pourtant compétent en matière de formation professionnelle des demandeurs d'emploi, le Conseil Régional n'a pas été cité parmi les principales parties prenantes de ce partenariat. Les instruments de formation professionnelle mobilisés pour répondre à l'objectif de cette cellule emploi LGV sont exclusivement nationaux (AFPA, Pôle Emploi, Direccte), laissant suggérer un pilotage centralisé, ou au mieux déconcentré, de la politique « emploi-formation » mise en œuvre.

Par ailleurs, la multiplicité des entreprises mobilisées pour la construction de cette LGV a nécessité une forte implication de Réseau Ferré de France, le maître d'ouvrage, dans la coordination du dispositif. Opérationnellement, la cellule emploi LGV s'était matérialisée par la mobilisation de conseillers de Pôle Emploi spécifiquement dédiés au projet LGV. Au plus fort de l'activité, treize personnels de Pôle Emploi travaillaient à temps plein sur le projet. A travers cette organisation est née la notion de «guichet unique » reprise, nous le verrons, dans le cadre de la construction de la LGV SEA. Finalement, la cellule emploi LGV a placé 3112 personnes sur la durée du chantier, dont 549 ont bénéficié d'une formation qualifiante. Comme ce fut le cas pour la gouvernance mise en œuvre dans le cadre de la construction du Tunnel sous la Manche, aucune évaluation indépendante n'a été conduite pour se prononcer sur l'efficacité du dispositif mis en œuvre. Il est certain que les besoins de main d'œuvre des entreprises impliquées dans la construction ont été satisfaits mais aucun élément ne permet de dire si les acteurs locaux ont bénéficié pleinement de cette organisation, et encore moins si celle-ci a eu un effet positif sur la

\footnotetext{
${ }^{6}$ Association nationale pour la formation professionnelle des adultes.

${ }^{7}$ Maison de l'information sur la formation et l'emploi.

${ }^{8}$ Plans locaux pluriannuels pour l'insertion et l'emploi.
} 
trajectoire professionnelle à moyen et long termes des personnes en ayant bénéficié.

Pour des projets futurs ou en cours, comme le Canal Seine Nord Europe ou encore le projet Lyon-Turin ferroviaire, des réflexions sont déjà en cours pour préparer la gouvernance des politiques «emploi/formation » pendant le chantier, ainsi que leur mutation après la construction. Ces réflexions se basent sur l'observation des modes de gouvernance à l'œuvre dans d'autres grands projets d'infrastructures de transport (Hasiak et al., 2007). Toutefois, ces observations souffrent du manque d'une véritable évaluation des processus à l'œuvre dans la construction et le pilotage de cette gouvernance, relativement aux buts qui leur étaient assignés. Dans cet article, nous proposons de dépasser ces limites en formulant un cadre d'analyse de la gouvernance « emploi-formation » dans le contexte de la construction de la LGV SEA Tours-Bordeaux pour la phase de recrutement ${ }^{9}$, dont l'objectif principal était la réponse aux besoins de main d'œuvre du constructeur. Ce travail s'inscrit plus largement dans les recommandations faites par des experts ayant étudié la relation entre grands chantiers et emploi local. Face aux difficultés de répondre à la question de l'effet des grands chantiers sur l'emploi local, Nadège Petitjean (2003) suggérait par exemple, pour des recherches futures, de mener une enquête auprès des agences d'intérim pour « comprendre comment s'organisent les politiques de recrutement et de formation mises en place par [les] acteurs et d'évaluer leur efficacité ».

\subsection{Méthodologie}

Pour mener à bien cette recherche au prisme d'une lecture proximiste, nous avons mobilisé quatre grands types de données qualitatives : i) des entretiens semi-directifs avec des «acteurs clés » du projet, ii) des documents officiels (conventions, contrats), iii) des articles de presse et iv) des notes de 12 réunions techniques et de pilotage auxquelles nous avons été associées.

Les entretiens semi-directifs ont été conduits entre mars 2013 et janvier 2014, à la fin de la phase de recrutement. Cinq acteurs institutionnels impliqués dans le système «emploi-formation » mis en œuvre ont été rencontrés. Au total, ce sont huit entretiens qui ont été conduits avec neuf personnes différentes. Ces entretiens semi-directifs avaient une visée exploratoire. Il s'agissait, grâce aux informations collectées, d'identifier le rôle de chaque acteur dans le processus de gouvernance « emploi-formation » et de reconstruire les étapes de mise en œuvre de celui-ci.

\footnotetext{
${ }^{9}$ Dans cet article, nous n'abordons pas, volontairement, la question de l'efficacité de la gouvernance à moyen terme de cette politique « emploi/formation » car, au moment de la rédaction de cet article, des recherches sont toujours en cours pour évaluer ses effets de moyen terme (pérennisation des emplois).
} 
Fig. 3 : Caractéristiques des entretiens semi-directifs

\begin{tabular}{|c|c|c|c|}
\hline Acteurs enquêtés & Nombre d'entretiens & Nombre d'individus & Fonctions \\
\hline COSEA & 4 & 2 & $\begin{array}{ll}\text { - } & \text { Directeur des } \\
& \text { ressources humaines } \\
\text { - } & \begin{array}{l}\text { Directeur de la } \\
\text { formation }\end{array}\end{array}$ \\
\hline $\begin{array}{l}\text { Pôle } \quad \text { Emploi } \\
\text { Poitou-Charentes }\end{array}$ & 1 & 2 & 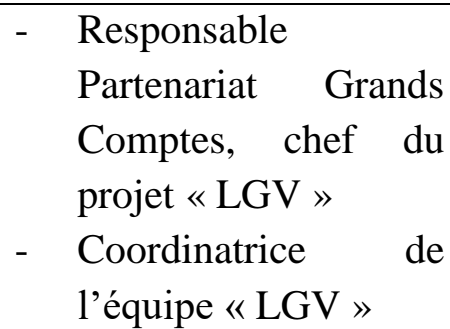 \\
\hline $\begin{array}{l}\text { DIRECCTE } \\
\text { Poitou-Charentes }\end{array}$ & 1 & 2 & 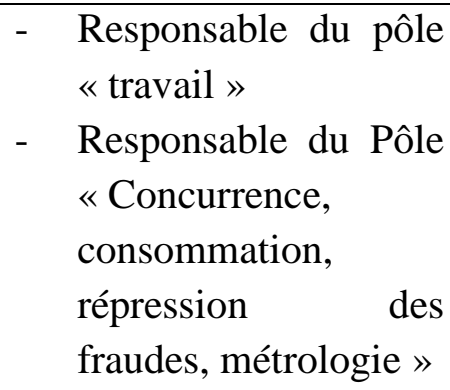 \\
\hline $\begin{array}{l}\text { Conseil Régional } \\
\text { Poitou-Charentes }\end{array}$ & 1 & 2 & $\begin{array}{l}\text { - Responsable de la } \\
\text { formation continue } \\
\text { - Chargée de mission } \\
\text { formation continue }\end{array}$ \\
\hline $\begin{array}{l}\text { Constructys } \\
\text { Poitou-Charentes }\end{array}$ & 1 & 1 & - Secrétaire générale \\
\hline
\end{tabular}

Quatre documents officiels ont été mobilisés pour déterminer le cadre institutionnel et légal du système de gouvernance. Les deux premiers sont des contrats : i) de concession entre SNCF Réseau (ex RFF) et LISEA, la société concessionnaire ii) de construction entre LISEA et COSEA, le constructeur. L'étude de ces deux contrats cherchait à interroger le caractère anticipé du système de gouvernance «emploi-formation" et à identifier l'échelle territoriale de son impulsion. Les deux autres documents officiels utilisés pour notre recherche sont des conventions de partenariat entre acteurs abordant les thématiques de l'emploi et de la formation. La première, signée en 2010 par l'État, le Conseil Général de la Charente, la Communauté d'agglomération du Grand Angoulême, la Communauté de communes de Cognac, des communes et EPCI, et SNCF Réseau, s'intitule "Charte de coopération territoriale en vue d'une réalisation exemplaire du chantier en matière de concertation, de facilitation et d'insertion ». La seconde convention de partenariat, signée en 2011, associe l'État, Pôle Emploi, COSEA et le Conseil Régional de Poitou-Charentes et se dénomme «Opération de construction de la LGV SEA ». L'analyse de ces conventions contribue à identifier les étapes de construction de la gouvernance de la politique 
« emploi-formation » du projet SEA, à repérer les engagements institutionnels des acteurs et leur contribution à la dynamique d'ensemble de la gouvernance.

Abordés avec précautions, les articles de presse apportent un double éclairage utile à la compréhension du système d'acteurs « emploi-formation ». D'une part, ils permettent de donner la parole à des acteurs peu écoutés ou sollicités dans le système de gouvernance. En ce sens, il contribue à apporter un regard critique à la politique mise en œuvre. D'autre part, ils rendent compte de la communication externe des acteurs institutionnels impliqués dans le système «emploi-formation », et donnent la possibilité de mettre en perspective les discours officiels avec la réalité des faits observés lors des entretiens semi-directifs et dans les documents officiels.

Enfin la participation aux 12 réunions techniques et de pilotage a permis de suivre le processus interne de gouvernance entre les acteurs et de repérer d'éventuelles particularités de fonctionnement qui n'auraient pas été mises au jour par l'intermédiaire des autres sources de données qualitatives.

2. Enjeux de la politique « emploi-formation » du projet LGV SEA et acteurs mobilisés

En 2012, COSEA, le groupement d'entreprises chargé de la conception et de la construction de la LGV SEA estimait à environ 5000 personnes la mobilisation nécessaire pour la phase «terrassement et génie civil». COSEA s'était aussi engagé à l'embauche locale de 1720 personnes entre mars 2012 et mars 2015 :

«La commande initiale était de passer de 500 à 5000 salariés en 6 à 8 mois [...] avec comme contrainte forte que deux tiers des salariés pouvaient être apportés par les associés du groupement et un tiers pouvait être des ressources trouvées localement. [...] Tout ça devait être processé, ça devait faire l'objet d'une démarche quasi-industrielle de sourcing [...]» (Entretien COSEA, Service RH, 20 mars 2013).

Pour estimer ce volume, le constructeur témoignait de son «savoir-faire » défini par une équation tenant compte de quatre variables: (i) les logiques financières (chiffre d'affaire); (ii) les différents jalons du chantier : la concertation, les études, les travaux d'infrastructure et de génie civil, les travaux d'équipements ferroviaires et les essais ; (iii) le contexte économique ; (iv) les enjeux environnementaux : les aléas climatiques et environnementaux (arrêtés lois sur l'eau, etc.) pouvant freiner le déroulement du chantier et le processus de recrutement ${ }^{10}$.

\footnotetext{
${ }^{10}$ En septembre 2012, la découverte d'une espèce d'amphibiens - le triton de blasius - a par exemple interrompu le chantier SEA et a eu un impact négatif sur l'emploi. Le respect des politiques environnementales aurait mis au chômage technique environ 1300 personnes (La Nouvelle République, 18/09/2012) Cet exemple n'est pas isolé. Entre 1996 et 2002, la construction de l'autoroute Alençon-Le Mans a été interrompue du fait de la présence d'un petit scarabée baptisé pique-prune.
} 
La phase de recrutement et de qualification des personnels a nécessité l'intervention de nombreux acteurs publics et privés, du niveau local à l'échelle nationale : l'État; le constructeur COSEA ; le concessionnaire LISEA; SNCF Réseau, le concédant; le Conseil Régional de PoitouCharentes ; Pôle Emploi Poitou-Charentes ; les Fédérations Nationale et Régional des Travaux Publics ou encore 1'Organisme Paritaire Collecteur Agréé (OPCA) Constructys. Il faut noter que si le projet SEA n'a pas bénéficié de la démarche «Grand chantier » de la part de l'État, on observe néanmoins que le groupement d'entreprises, COSEA, s'est approprié ce dispositif pour le mettre en œuvre dans le cadre de son chantier. Nous le verrons, ce positionnement nouveau modifie les rapports entre acteurs publics et privés et plus largement la gouvernance territoriale.

L'ensemble de ces acteurs s'accordent sur un objectif: le recrutement pour une période non déterminée lors de la phase «Terrassement et Génie Civil »d'environ 1500 salariés locaux dont une partie en situation d'insertion. Toutefois, des intérêts particuliers qui sont autant de sources potentielles de coopérations ou de conflits demeurent. En effet, ces intérêts supposent des postures d'acteurs qui vont influencer la coordination et dessiner les relations entre acteurs. De ces fondements et de leur bonne articulation dépend la réussite de la phase de recrutement.

Pour COSEA, la construction de la LGV SEA, par l'ampleur du projet, a nécessité la mise en place d'une politique de ressources humaines hors normes, avec en fond la priorité de répondre à ses propres intérêts. (i) L'intérêt premier du constructeur est de réaliser les recrutements au moindre coût. En effet, le coût de la construction étant forfaitaire, aucun dépassement par le constructeur n'est compensé par les financeurs. La coopération avec les acteurs locaux vise pour COSEA à limiter ses coûts de transaction par un recrutement de personnels locaux :

Il fallait constituer un vivier de Tours à Bordeaux. On a mis en place avec Pôle Emploi et les organismes de formation des informations collectives au plus près des communes. L'important, c'est la proximité entre le domicile et le lieu de formation et d'emploi. C'est pour ça que le vivier n'est constitué que de personnes vivant prêt de la trace. Toutes les personnes qui étaient intéressées ont eu un entretien individuel. [...] il y a eu quasiment 4000 personnes convoquées, plus de 2500 présents, 2200 entretiens individuels. Tout ça était fait par Pôle Emploi, les responsables RH de section et on s'est constitué un vivier de plus de 2000 candidats potentiels [...] (Entretien COSEA, Service des RH, 20 mars 2013).

La mise en œuvre de cette démarche de recrutement à très grande échelle a nécessité l'enchaînement de plusieurs étapes :

"Pour faire en sorte que le langage soit commun, fin 2010 - début 2011, on a organisé des bus qui étaient occupés par l'ensemble des prescripteurs qui peuvent nous amener des ressources et on leur a dit : "demain quand on va vous demander un compacteur, un coffreur, etc. voilà ce sera ce métier-là ». Ensuite la commande est devenue claire. Il fallait constituer un vivier de Tours à Bordeaux. Pour faire cela, on a mis en place avec Pôle Emploi et les organismes de formation des informations collectives au plus près des communes (des plus grosses au plus petites). Ces 
informations collectives ne concernaient que les demandeurs d'emploi, c'était sur convocation de Pôle Emploi. Toutes les personnes qui étaient intéressées ont eu un entretien individuel » (Entretien COSEA, Service RH, 20 mars 2013).

Cette démarche est d'autant plus intéressante pour le constructeur que le secteur des travaux publics se caractérise par des primes de grand déplacement octroyées aux salariés déplacés. Afin de minimiser ces primes, qui peuvent atteindre $1000 €$ mensuels pour un ouvrier (Lempereur $\&$ Fouqueray, 2014), les entreprises de BTP sont davantage tentées de s'orienter vers la main d'œuvre locale, moins chère, autant, voire plus productive, et dont le recrutement par contrat à durée de chantier n'engage pas l'entreprise au-delà du projet. (ii) Le deuxième intérêt, qui doit participer à répondre au premier, réside dans la constitution d'un partenariat avec les acteurs locaux pour identifier, informer et convoquer les ressources humaines locales disponibles (sourcing). COSEA $^{11}$ est un constructeur éphémère qui est né avec le projet SEA et qui disparaitra lorsque la construction sera terminée. Ancrée localement, compte tenu de sa localisation, cette firme est aussi et avant tout nationale voire internationale car rattachée à plusieurs grands groupes de travaux publics (Fig. 4).

\footnotetext{
${ }^{11}$ Ce groupement est conduit par VINCI Construction (Vinci Construction Terrassement, Vinci Construction France, Vinci Construction Grands Projets, Dodin Campenon Bernard), et composé également d'Eurovia Infra, d'ETF, du pôle Énergies de VINCI, et de Inéo Rail et Systra. Piloté et coordonné par la Direction de Projet (DPR), COSEA s'organise autour de 5 sous-groupements d'entreprises qui réunissent l'ensemble des compétences nécessaires à la construction de la ligne à grande vitesse :

- le Sous-Groupement Conception (SGC) : conception de la ligne.

- le Sous-Groupement Infrastructures (SGI) : génie civil.

- le Sous-Groupement Superstructures (SGS) : bases travaux ferroviaires, voies ferroviaires et caténaires.

- le Sous-Groupement Signalisation-Télécommunications (SGST) : signalisation, télécommunications, basse tension et contrôle d'accès.

- le Sous-Groupement Énergie (SGE) : travaux d'alimentation électrique.
} 
Fig. 4 : Schéma de 1’organisation du groupement COSEA (source : Vinci retravaillé)

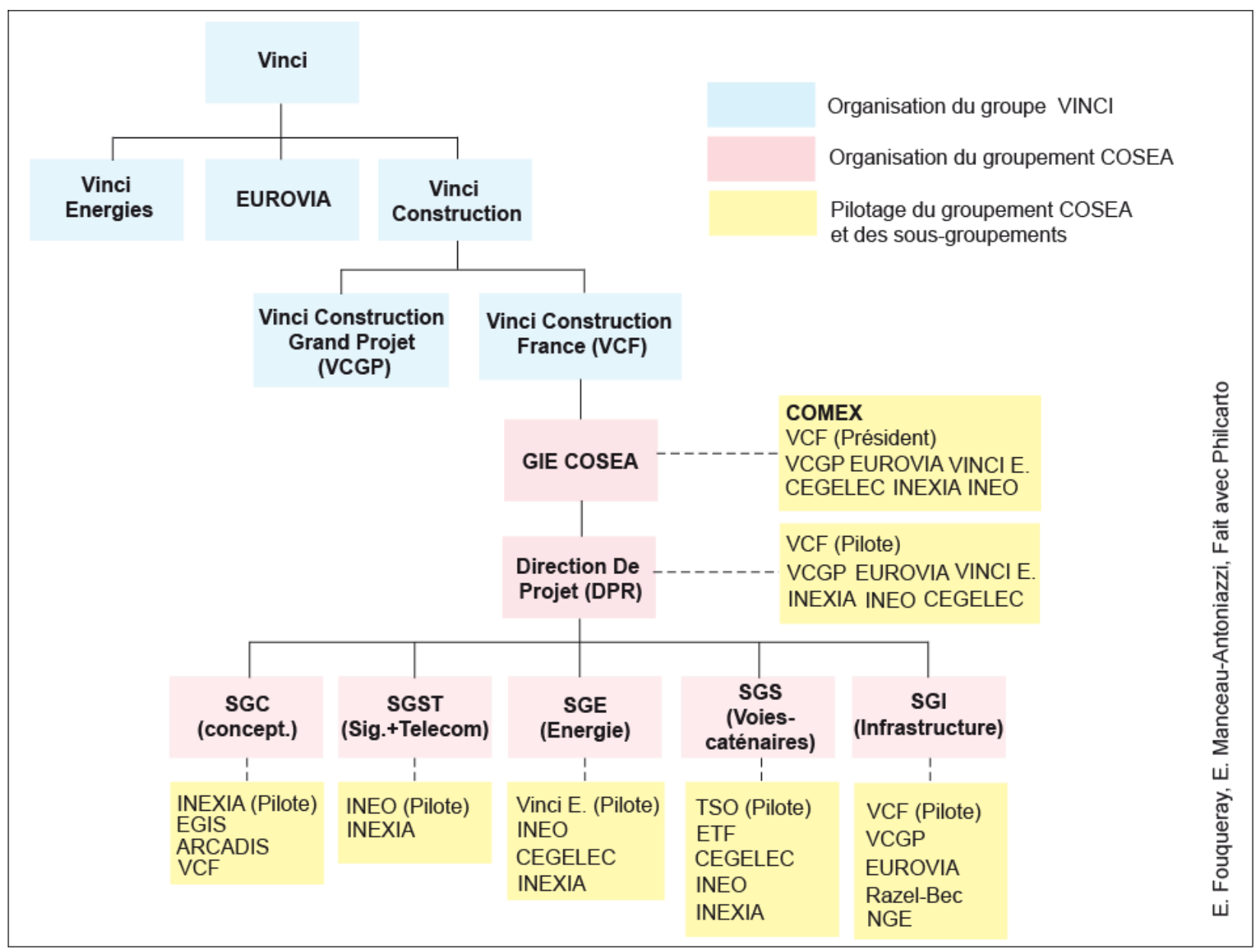

Cette double inscription spatiale n'est pas sans incidence sur la stratégie du constructeur vis-à-vis des ressources humaines locales que l'on peut qualifier de combinatoire car elle vise à associer, à des ressources internes extérieures au territoire, des ressources locales (Gilly et Perrat, 2003). En effet, la firme noue des liens importants avec le tissu économique local afin d'assurer une complémentarité entre ses ressources propres et les ressources disponibles sur le territoire. (iii) Enfin, le dernier intérêt pour le constructeur est celui de l'image. Un tel projet peut contribuer à valoriser ou dévaloriser l'image du groupe qui en supporte la charge. L'acceptation par la population et les institutions locales du projet passe donc par l'inscription territoriale du constructeur, dont l'action en faveur de l'emploi local est le meilleur atout :

"J'avais conscience qu'il fallait qu'on s'intègre territorialement (retombées en termes de recrutements) alors que si on avait laissé faire comme d'habitude, très majoritairement il y aurait eu du recours à l'intérim avec des entreprises de travail temporaire qui seraient venues de partout. Et donc ça on ne l'a pas voulu (Entretien COSEA, Service RH, 20 mars 2013). 
Le constructeur doit aussi rendre des comptes sur l'avancement de la construction à plusieurs acteurs clés : L’État, le concessionnaire de la LGV, le concédant et la Région Poitou-Charentes. L'État, à travers la préfecture de la région Poitou-Charentes, coordonne le chantier sur les aspects environnementaux et la politique de l'emploi. Cette politique de l'emploi s'est caractérisée par la mise en place d'un guichet unique sous l'impulsion du préfet. A travers la politique de l'emploi, l'État cherche à s'assurer de l'acceptation du projet par les acteurs locaux, notamment les financeurs, et à garantir l'avancement du chantier et sa soutenabilité économique inscrits dans les contrats passés entre le concédant et le concessionnaire. L'objectif est aussi de maximiser les retombées économiques du projet de construction, et notamment l'effet de relance économique que peut avoir un tel investissement.

La mise en œuvre de la coordination «emploi-formation» ne représente pas pour le concessionnaire, LISEA, un enjeu particulier. Celui-ci s'attache davantage à veiller aux respects des plannings prévisionnels et du coût prévisionnel des travaux. COSEA et LISEA appartenant au même groupe, ces sociétés partagent l'intérêt commun que le projet soit économiquement rentable. On peut toutefois souligner que cette coordination contribue à l'image du projet LGV SEA et du groupe Vinci sur les territoires traversés et que de fait, si elle permet de la valoriser, l'action mérite d'être soutenue.

Du point de vue du concédant, SNCF Réseau, l'intérêt est plus marqué. Pour ce dernier, ce n'est pas tant le fait qu'il soit favorable, a priori, à une stratégie de ressources humaines plutôt qu'à une autre. Son attente est fortement influencée par le mode de financement du projet. Les collectivités locales traversées par la ligne contribuent à plus d'un milliard d'euros au financement du projet. Certaines ne verront que les trains passer et attendent en retour de leur contribution des retombées économiques liées à la construction, notamment en matière d'emploi. Le concédant est donc incité à encourager toute initiative du constructeur en faveur de retombées économiques positives locales.

Le Conseil Régional de Poitou-Charentes a également voix au chapitre. Si la région a décidé de ne pas financer la construction de la ligne, estimant qu'il s'agissait avant tout d'un projet d'envergure nationale voire européenne, elle est la seule des trois régions traversées à avoir financé des formations spécifiques au projet LGV SEA. Son objectif est de « doter chacun d'une qualification professionnelle reconnue sur le marché du travail» (Technicien du Conseil Régional Poitou-Charentes). Ne pouvant afficher qu'elle ne soutient que les formations du projet LGV SEA, la région a fait le choix de créer une politique de soutien aux grands chantiers dont la LGV fait partie.

Autre acteur incontournable de l'emploi et de la formation, Pôle Emploi Poitou-Charentes et plus largement Pôle Emploi national sont impliqués dans la coordination ${ }^{12}$. Leur participation à cette

${ }^{12}$ Pôle Emploi intervient dans le financement des indemnités de chômage et dans celui des formations professionnelles. 
politique locale s'inscrit dans leur mission de service public dont l'objectif est de « garantir l'accompagnement des demandeurs d'emploi dans leur recherche d'emploi et à répondre aux besoins de recrutement des entreprises » (Pôle Emploi, 2015). L'intérêt pour Pôle Emploi de participer à cette coordination est d'élargir les potentiels d'emplois pour les demandeurs d'emplois de Poitou-Charentes. Le chantier représente une opportunité pour l'institution d'insérer ou de réinsérer des personnes plus ou moins éloignées de l'emploi.

Deux autres acteurs parties prenantes représentent la branche de la construction et plus particulièrement celle des Travaux Publics. Il s'agit de la Fédération Nationale des Travaux Publics, qui est présente en Poitou-Charentes sous l'égide de la Fédération Régionale des Travaux Publics de Poitou-Charentes et de l'OPCA Constructys, qui intervient également à l'échelle régionale. La participation de ces acteurs à la coordination a été impulsée au niveau national. Le lobbying du groupe Vinci et des associés à COSEA a permis leur adhésion au projet et leur soutien pour le financement de formations professionnelles. Ces acteurs attendent, en retour de leur contribution, que les personnes dont ils financent les formations spécifiquement pour le projet SEA soient à terme embauchées par Vinci.

L'ensemble de ces acteurs ont contribué à la mise en place de la gouvernance locale autour du projet de la LGV SEA par le biais de proximités institutionnelle et organisationnelle. Mais cette mobilisation des acteurs s'est également traduite par une proximité en termes de relations sociales, ciment de la réussite de la phase de recrutement du chantier.

3. Les fondements de la gouvernance locale «emploi-formation » du projet LGV SEA : proximités institutionnelle et organisationnelle et relations sociales

A partir du cadre théorique présenté dans la première partie et de l'analyse du positionnement de chaque acteur (en partie 2), nous analysons les trois socles de la gouvernance locale du dispositif «emploi-formation» de la construction de la LGV SEA, constitués par la proximité institutionnelle, la proximité organisationnelle et les relations sociales. Nous ne revenons pas ici sur la proximité géographique, puisque la LGV SEA et les acteurs mobilisés dans la politique «emploi-formation » sont situés en région Poitou-Charentes et cette localisation est le facteur déclenchant de leur engagement dans celle-ci.

(i) Le dispositif institutionnel visant à favoriser le recrutement de salariés locaux sur le chantier a été amorcé dès 2010 à travers une « charte de coopération territoriale en vue d'une réalisation exemplaire du chantier en matière de concertation, de facilitation et d'insertion » signée entre l'État, plusieurs collectivités territoriales ${ }^{13}$ traversées par la ligne et bien entendu SNCF Réseau, à l'initiative. Parallèlement, le groupement LISEA composé du groupe Vinci, de la Caisse des

\footnotetext{
${ }^{13}$ Le Conseil Général de la Charente, la Communauté d'agglomération du Grand Angoulême, la Communauté de communes de Cognac, les Communes et les EPCI signataires.
} 
dépôts et d'Axa était déclaré concessionnaire pressenti, ce qui autorisait le début des négociations avec RFF (Coux, 2012). COSEA n'est logiquement pas signataire de cette première charte puisque cette dernière est antérieure à la création du groupement. S'appuyant sur cet héritage institutionnel impulsé par le concédant, le constructeur et les acteurs de l'emploi et de la formation ont ensuite élaboré des règles du jeu plus précises pour encadrer la gouvernance et la coopération entre les acteurs. Celles-ci ont pris la forme de plusieurs accords-cadres rédigés et signés au fur et à mesure du projet. En 2011, un premier partenariat entre l'État, Pôle Emploi, la Région Poitou-Charentes et cette fois-ci COSEA est établi. Cet accord-cadre visait le bon déroulement du partenariat face aux enjeux soulevés par le projet de la LGV SEA :

"Afin de répondre à cet enjeu [l'embauche de salariés locaux, avec un engagement de consacrer $10 \%$ du volume d'heures de génie civil et de terrassement aux publics en difficulté d'insertion sociale et professionnelle], les signataires de la présente convention entendent agir en concertation pour répondre aux besoins de main-d'œuvre qualifiée exprimés par le constructeur de la ligne $L G V$, dans les meilleurs conditions et avec l'appui des compétences locales en termes de recrutement, de formation initiale et continue, d'insertion, dans une dynamique de développement économique et de sécurisation des parcours»

Cet accord cadre a ensuite été décliné en Gironde et en Indre-et-Loire, ainsi qu'à l'échelle du département de la Charente, déjà signataire de la charte de coopération territoriale. Ces conventions de partenariat matérialisent une proximité institutionnelle d'adhésion entre les acteurs (Pecqueur \& Zimmermann, 2004).

Au regard des intérêts divergents des acteurs signataires de ces conventions, nous observons que cette proximité institutionnelle d'adhésion s'inscrit « dans un rapport contradictoire de pouvoirs » entre acteurs privés (logique de profit) et publics (logique de bien public) (Gilly \& Perrat, 2003). Du fait de l'absence d'antériorité dans les rapports entre le constructeur et les autres acteurs, la construction de cette proximité institutionnelle a nécessité au préalable de multiplier les interactions en face à face «pour réduire l'incertitude et faire émerger une situation de confiance ou de défiance » (Ferrary, 2010, p.189). Cette stratégie volontaire d' «apprentissage social» permet de réduire le risque d'échec de la coordination et s'apparente à un investissement pour le constructeur (ibid.). De nombreux travaux soulignent le rôle considérable que jouent les relations sociales interindividuelles lorsque les acteurs ne se connaissent pas préalablement (Dupuy \& Torre, 2004; Granovetter, 1985; Grossetti, 2004; Raveyre, 2005b). L'analyse du processus de gouvernance du dispositif «emploi-formation» met en exergue l'importance des relations sociales dans sa construction.

(ii) Dans l'objectif d'instaurer une confiance avec les acteurs locaux, en particulier avec ceux avec qui il n'avait pas de pouvoir de négociation (Conseil Régional, Pôle Emploi), le constructeur s'est engagé dans une stratégie de développement des relations sociales interpersonnelles et interorganisationnelles : 
«Dans tous les sujets, les mises en place passent par les hommes et les femmes et donc il faut connaître les gens. A partir de l'été 2010, j'ai eu une quantité de réunions avec Pôle Emploi, l'État et le Conseil Régional : il fallait que l'on apprenne à se connaître » (Entretien COSEA, Service RH, 20 mars 2013).

On peut supposer qu'à l'origine, les acteurs locaux de l'emploi et de la formation n'avaient pas connaissance de la stratégie de Vinci pour approvisionner en main d'œuvre le chantier. Pour ce faire et avec l'objectif de faciliter ses relations avec les institutions locales, COSEA a recruté un professionnel local de l'emploi et de la formation au poste de directeur de l'insertion et de la formation. En effet, les relations sociales interpersonnelles préexistantes entre ce directeur et les techniciens des institutions partenaires (Pôle Emploi et Conseil Régional) ont permis d'assurer les coopérations inter-organisationnelles. «La confiance organisationnelle constitue une extension de la relation de confiance interpersonnelle (relation de face-à-face) au principe de l'action collective » (Dupuy \& Torre, 2004, p.83). Cet encastrement du responsable de la formation de COSEA au sein des réseaux locaux de l'emploi et de la formation découle d'une véritable stratégie du constructeur de créer une relation de confiance avec ses partenaires « clés ». Le résultat a été au rendez-vous :

«Ce qui m'a toujours rassuré, c'est qu'il y avait [Monsieur X]. Et [Monsieur X] dans le jeu, c'est bien. C'est un type bien, on peut lui faire confiance. C'est un type fiable. C'est une vraie caution morale » (Entretien Conseil Régional Poitou-Charentes, Service Formation, 25 mars 2013).

Cette relation sociale interpersonnelle a été le ciment de l'instauration d'une «confiance en COSEA ». Comme se plaît à le dire un responsable du constructeur : «je suis copain avec tout le monde. Je connais tout le monde », ce qui souligne cette stratégie de création d'une confiance par les relations sociales.

Le développement d'une confiance réciproque entre les partenaires à travers le dialogue et les relations sociales a permis d'engager le travail commun autour du projet de recrutement de personnels locaux :

On a d'abord appris à se connaître et ensuite à avoir le même langage. C'est d'ailleurs après qu'on a écrit nos conventions partenariales : on a décrit ce qu'on faisait plutôt que d'écrire ce qu'on allait faire [...] J'aurais pu imaginer un dispositif où les groupements d'associés, Vinci en tête, étaient auto-suffisants, qui aurait pu se substituer à Pôle Emploi [...] mais ce choix aurait été complètement inutile. Il n'est pas question de payer deux fois. Pôle Emploi, on le paye déjà dans nos impôts. Si j'avais mis une structure en place, on payait deux fois. [...] On va considérer que c'est éventuellement un financement masqué, on peut dire qu'à la limite si Pôle Emploi ne l'avait pas fait nous aurions dû mettre des structures au-delà de nos structures RH. Quelque part, c'est une manière de financer notre sourcing. Et l'autre financement qui a été fait par Pôle Emploi, et qui 
n'est pas neutre, c'est le financement des préparations opérationnelles à l'emploi en partenariat avec Constructys (Entretien COSEA, Service RH, 20 mars 2013).

Bien avant que les recrutements ne débutent, des comités techniques ont eu lieu tous les mois dès 2010. Tenant compte des contraintes et des intérêts des uns et des autres, les acteurs se sont donc mis d'accord sur le rôle de chacun :

"On s'est vite retrouvé autour d'une table avec COSEA, l'État et Pôle Emploi pour accompagner le développement de cette $L G V$ : on a bâti un protocole d'accord entre nous sur qui fait quoi. On s'est appuyé sur le modèle de la LGV Rhin-Rhône où les pouvoirs publics se sont retrouvés autour de la table » (Entretien Conseil Régional Poitou-Charentes, Service Formation, 25 mars 2013).

"A la fois, les masques sont tombés et on a réussi à se connaître, à se comprendre, à connaître les contraintes des uns et des autres, et un peu à la fois on a écrit et imaginé comment on allait travailler avec chacun dans un rôle bien précis : Pôle Emploi en termes de sourcing, le conseil régional en termes de formation professionnelle, et la Direccte pour sécuriser tous les aspects réglementaires à l'occasion de ces recrutements » (Entretien COSEA, Service RH, 20 mars 2013).

D'un point de vue organisationnel, le partenariat mis en place sous l'impulsion de l'État s'est accompagné de la création d'une Cellule LGV chez Pôle Emploi caractérisée par le système de guichet unique dédié au recrutement LGV et du financement de programmes de formations régionaux spécifiques LGV associant Pôle Emploi, le Conseil Régional, l'OPCA Constructys et le constructeur COSEA. Par ailleurs, les acteurs (Conseil Régional, État, Pôle Emploi, COSEA) se sont rencontrés tous les 2 à 3 mois dans le cadre d'un comité technique permettant de guider l'action.

"Il s'agissait de travailler avec Pôle Emploi en tant que guichet unique : toutes les ressources s'adressent à Pôle Emploi et nous, on dépose toutes nos offres à Pôle Emploi, c'est une espèce de passage obligé [...] On ne discutait qu'avec la direction régionale de Poitou-Charentes, après c'était à eux de mettre en place. C'est toujours l'esprit de guichet unique, moi j'aime pas les réunions forums, j'aime bien une tête, je m'adresse à une personne et puis voilà. »(Entretien COSEA, Service RH, 20 mars 2013).

Pôle Emploi a dédié une équipe de conseillers spécifiquement au projet SEA, située dès l'origine dans les locaux de COSEA à la demande de la direction des Ressources Humaines du groupement. Au plus fort de l'activité de recrutement, huit membres du personnel de Pôle Emploi travaillaient pour le projet SEA. Cette «internalisation» de Pôle Emploi par le constructeur traduit le glissement d'une proximité institutionnelle vers une véritable proximité organisationnelle, motrice du système de gouvernance public/privé mis en œuvre et hérité, on peut le supposer, des expériences précédentes. 
Fig. 5 : Des accords-cadres à l'instauration de la confiance. Le processus d'élaboration de la coordination «emploi-formation »

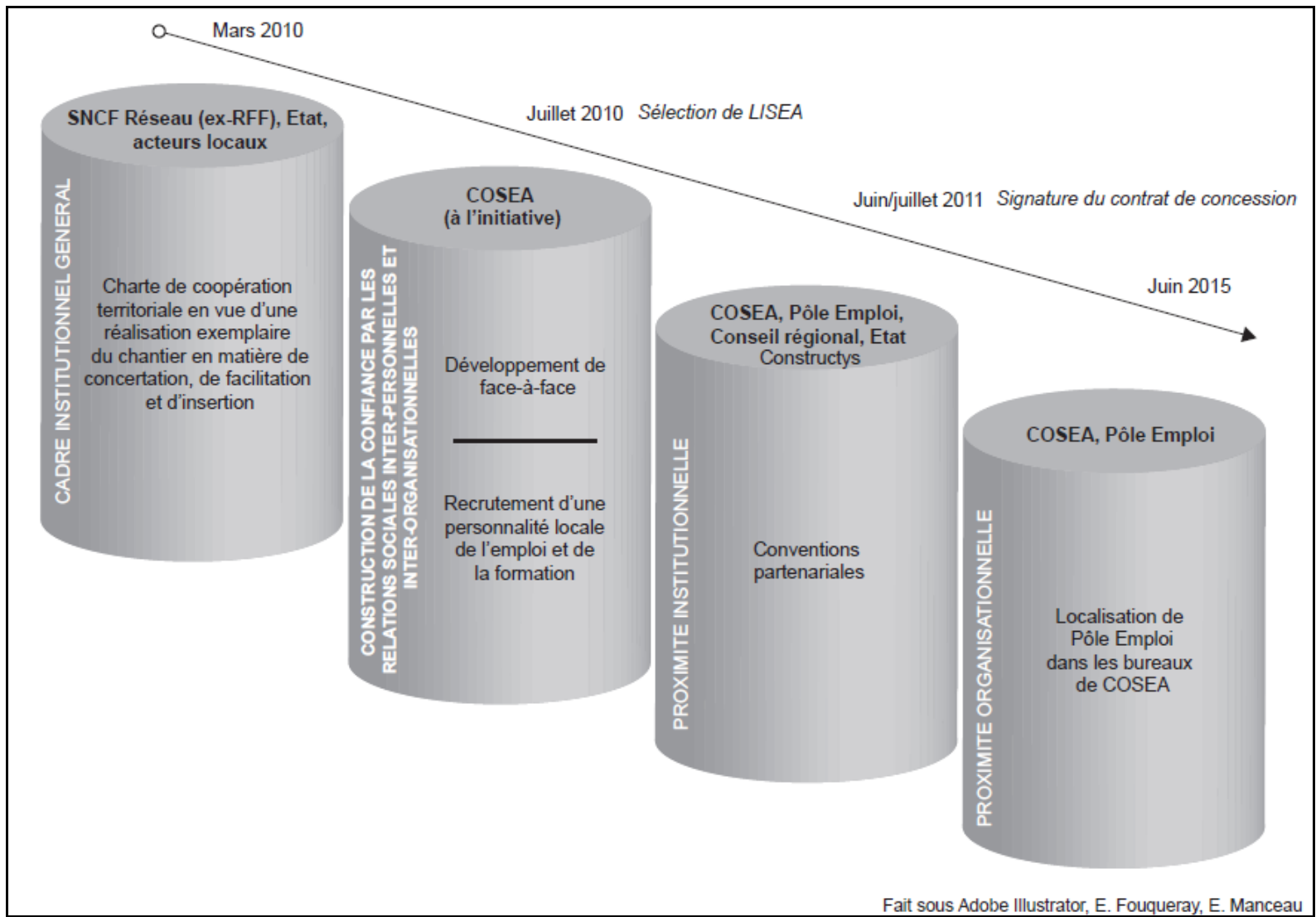

Au plus fort du chantier à l'été 2013, le projet a finalement mobilisé 9000 personnes, soit 4000 personnes de plus que ce qui était prévu. Après s'être maintenus à un niveau élevé jusqu'à la fin de l'été 2014, les effectifs du chantier ont connu une décrue jusqu'au printemps 2015. Une évaluation des retombées économiques de la construction de la ligne pour les régions Aquitaine, Poitou-Charentes et Centre fait état d'une création de richesse de 755 millions d'euros sur la période 2011 - 2013, soit 13799 emplois directs, indirects et induits (en Equivalent Temps Plein Annuel) (Fouqueray, 2016). 
Fig. 6 : Les chiffres de l'emploi en $\mathrm{ETP}^{14}$ du chantier SEA pour la période 03/2010-01/2015

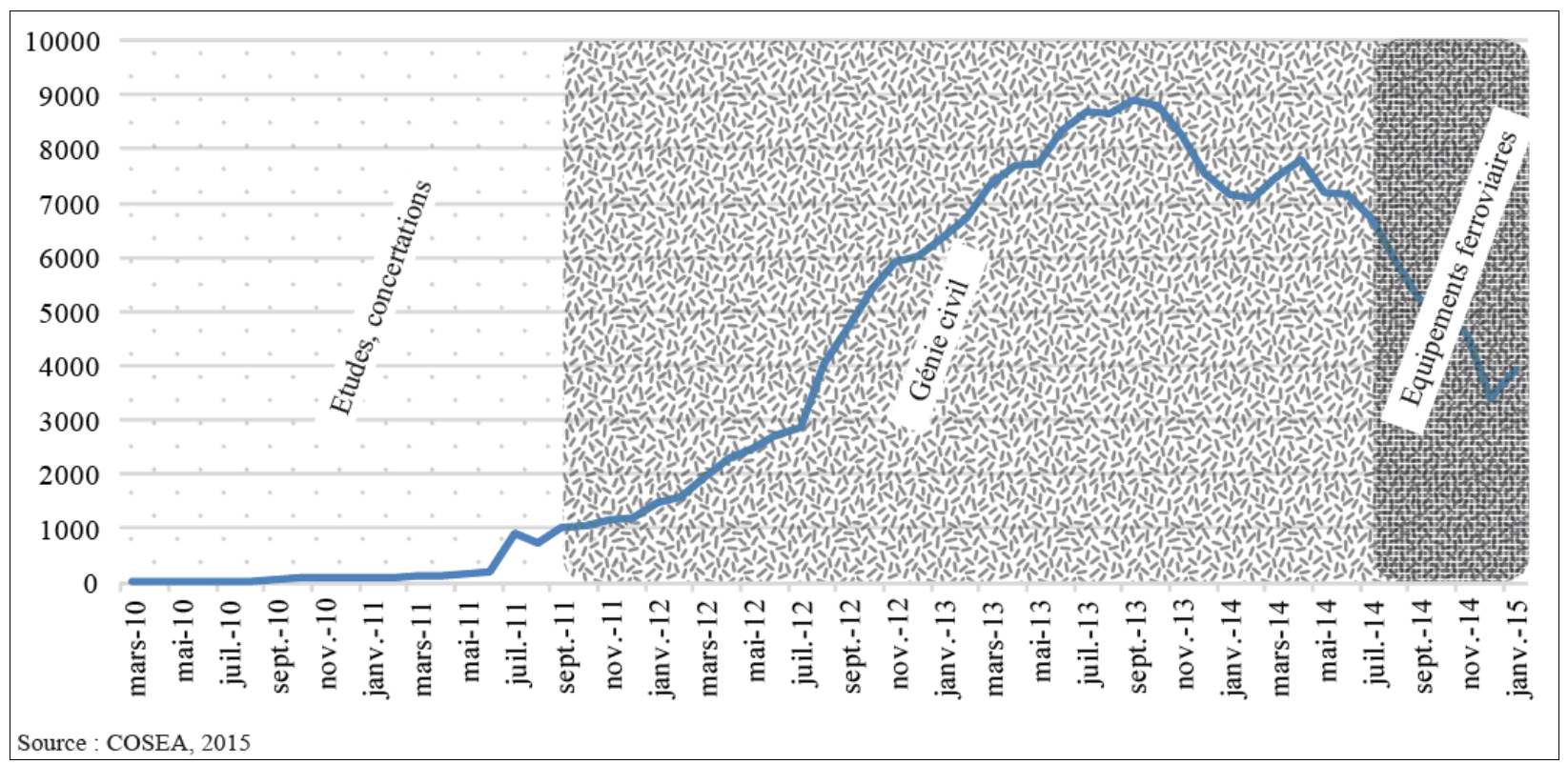

Les entreprises associées ont apporté trois quarts des besoins de main d'œuvre en affectant des personnels issus de leurs différentes entités et 2000 personnes ont été embauchées dans les 6 départements traversés par la ligne ${ }^{15}$, dont 1400 dans le cadre de l'insertion professionnelle ${ }^{16}$ (COSEA). Le dispositif aurait par ailleurs permis, selon les chiffres présentés par la plateforme d'appui aux mutations économiques de la LGV SEA, labélisée par l'État et issu du Pacte national pour la Croissance, la compétitivité et l'emploi ${ }^{17}$, la réalisation au printemps 2015 de 1300000 heures de travail d'insertion.

\footnotetext{
${ }^{14}$ ETP ou équivalent temps plein : unité de décompte dans laquelle sont exprimés à la fois les plafonds d'emplois et les consommations de ces plafonds.

${ }^{15}$ La multiplicité des chiffres annoncés par le constructeur COSEA, Pôle Emploi ou encore la préfecture de région peut rendre difficile la lecture des résultats dans la phase des recrutements locaux et dans la phase de redéploiement des salariés. Selon les moments et les interlocuteurs, entre 1300 et 2000 emplois locaux ont été annoncés.

16 La définition de l'insertion professionnelle retenue par le constructeur COSEA concerne les personnes bénéficiaires des minimas sociaux (RSA, ASS), jeunes sans qualifications de moins de 26 ans, personnes relevant de l'obligation d'emploi, personnes de plus de cinquante ans, demandeurs d'emploi de plus d'un an et les femmes.

${ }^{17}$ Le Pacte national pour la Croissance, la Compétitivité et l'Emploi mis en œuvre par le gouvernement en novembre 2012 a été décliné en huit leviers de compétitivité et 35 mesures. Il prévoit notamment la création de 13 plateformes d'appui aux mutations économiques mises en place dans des territoires sur le plan national (mesure 21). Ces plateformes s'articulent autour de démarches de soutien à l'anticipation et à la gestion des ressources humaines afin d'accompagner les entreprises, TPE ou PME, et pour assurer la sécurisation des parcours professionnels des salariés. Les territoires traversés par la LGV SEA constituent l'une de ces 13 plateformes.
} 
Fig. 7 : D’où viennent les salariés du chantier de la LGV SEA $?^{18}$

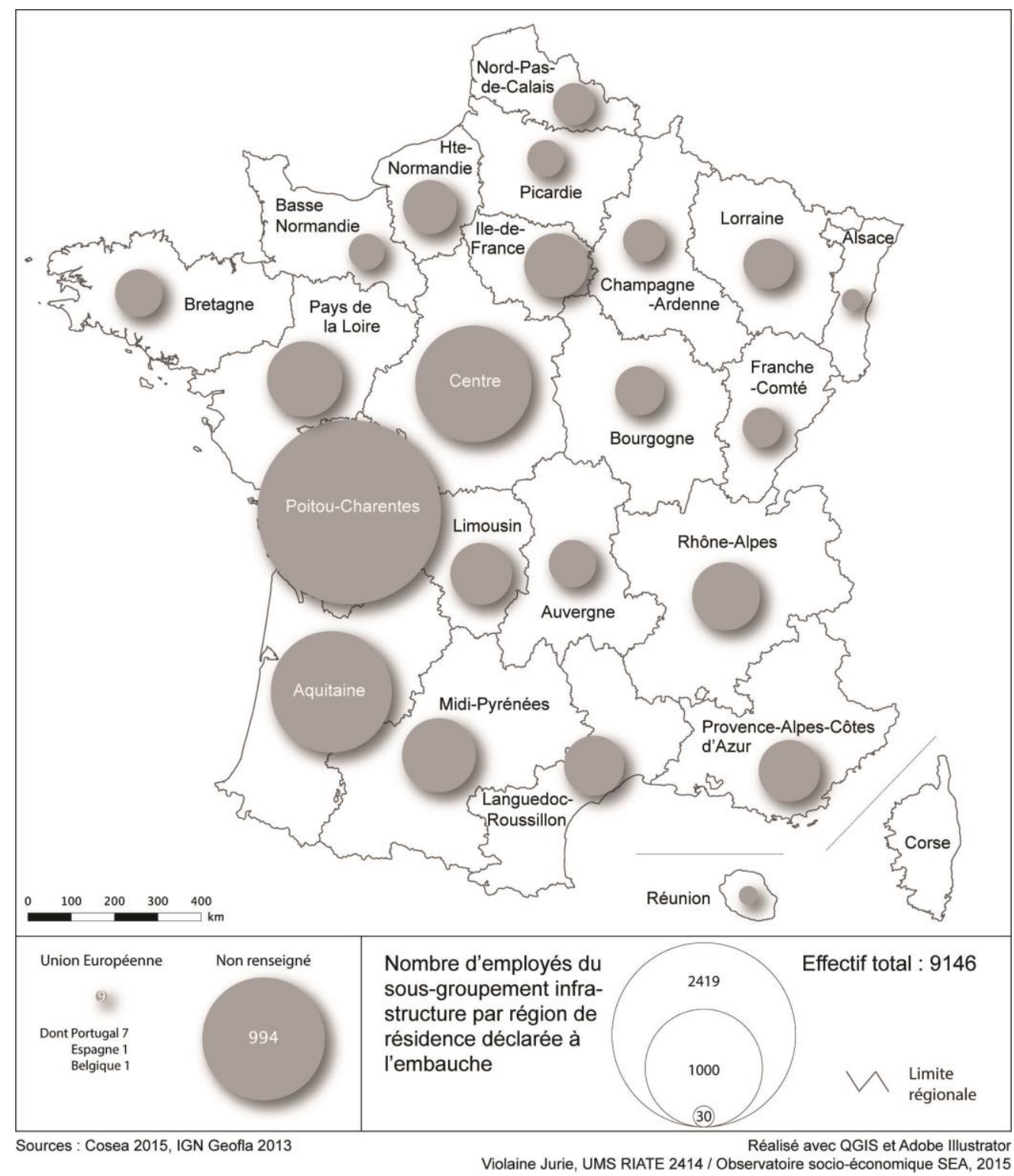

En fonction de la place occupée par les acteurs, les retours d'expériences sur le dispositif mis en œuvre pour la phase de recrutement diffèrent toutefois sensiblement. Alors que les acteurs clés (COSEA, Pôle Emploi, Conseil Régional) en ont une opinion positive, le point de vue des acteurs secondaires est plus contrasté.

Ainsi, le constructeur considère que sur le territoire, de Tours à Bordeaux, tous les engagements pris sont tenus, voire dépassés. Cette réussite est le fruit, selon lui, de la coordination entre les

${ }^{18}$ La carte représente le nombre d'employés du Sous-Groupement Infrastructure par région de résidence déclarée à l'embauche. Elle ne permet pas d'identifier l'origine des salariés. On peut supposer que les "non renseigné" représentent le lieu de résidence à l'étranger d'une partie des compagnons présent sur le chantier. 
acteurs publics et privés. Elle s'inscrit également dans une qualité des relations interpersonnelles entre décideurs des différents partenaires :

«Pôle Emploi a fait un travail formidable. En plus, on est devenu ami, copain, on se voit, on s'embrasse, [Monsieur Y] c'est un copain. Et quand c'est comme ça tout devient simple [...] L'entreprise seule n'aurait pas su faire, les pouvoirs publics seuls n'auraient pas pu le faire mais quand public et privé arrivent à se retrouver, à se connaître, à jouer en équipe sur des missions comme ça d'intérêt général, public et privé ça fait des choses formidables » (Entretien COSEA, Service RH, 20 mars 2013).

Les entretiens menés avec des personnels de Pôle Emploi confirment l'appréciation du constructeur concernant la coordination mise en place, avec plus de mesure toutefois :

"On travaille bien et surtout par anticipation. On travaille de façon collégiale » (Entretien Pôle Emploi, Cellule LGV SEA, 29 mars 2013).

Les techniciens du Conseil Régional en charge du projet soulignent également la qualité du travail effectué et la pertinence du système organisationnel mis en œuvre. Quelques doutes et regrets sont tout de même affichés quant aux résultats du dispositif en termes de taux de recrutements, liés notamment au fait que les entreprises associées dans le groupement ne jouaient pas toutes le jeu du recrutement local de demandeurs d'emplois qualifiés ${ }^{19}$ :

"Le schéma de démarrage était bien pensé avec une cellule de Pôle Emploi avec qui le Conseil Régional a très bien travaillé [...] Au démarrage, on a quelque chose qui est très maillé et qui offre un réel parcours aux demandeurs d'emploi. Après dans la réalité, tout n'est pas atteint... On a un peu déchanté» (Entretien Conseil Régional de Poitou-Charentes, Service Formation, 25 mars 2013).

Les acteurs secondaires sont plus nuancés sur le dispositif mis en œuvre pour la phase de recrutements. L'OPCA Constructys considère que les institutionnels (État, Région) ont été suffisamment associés au projet, tandis que «la CCI et la Fédération Régionale des Travaux Publics ont été mobilisés car on avait besoin d'eux ». L'implication de l'OPCA Constructys en Poitou-Charentes découle d'une décision nationale de la Fédération Nationale des Travaux Publics. Cette dernière a passé un accord avec Constructys national. "Ensuite toutes les conventions se sont faites au niveau régional»(Constructys Poitou-Charentes). Constructys Poitou-Charentes n'a donc pas été moteur dans la collaboration. Il a subi des décisions nationales ce qui le place dans une position assez critique vis-à-vis de COSEA :

"Tout le monde est reparti en trainant la patte : investissement énorme et peu de retours et de recrutements pérennes, on forme beaucoup et COSEA fait revenir des salariés [...] Le projet a détruit de l'emploi, car des professionnels ont quitté des entreprises locales attirés par Vinci, les

${ }^{19} \mathrm{Au}$ moment où ont eu lieu ces entretiens, ces recrutements n’étaient pas terminés. 
entreprises locales ont perdu des compétences» (Entretien Constructys Poitou-Charentes, Secrétariat général, 19 avril 2013).

Un technicien de la Direccte Poitou-Charentes mobilisé sur le projet SEA a également un regard critique sur le système «emploi-formation ». Ce service déconcentré de l'État avait un rôle de contrôle de la démarche mise en œuvre et n'était pas pilote :

"Très bien pour répondre aux besoins de l'entreprise tout en veillant à faciliter l'insertion de publics en difficulté. Ok, ça a marché! Pour le reste, la qualité du travail, la qualité de l'emploi, l'accueil (hébergement), etc....il y aurait pu y avoir, notamment dans le cadre d'une GPEC territoriale, quelque chose d'un peu plus dynamique... Il y a eu également des promesses non tenues [...] Au départ COSEA avait dit : "mais les grands déplacements ça nous coûte les yeux de la tête », et à mon avis finalement, les entreprises du groupe sont venues avec leurs salariés car ils n'avaient pas d'autres boulots à leur faire faire, ils ont payé les grands déplacements, ils sont là. » (Entretien Direccte Poitou-Charentes, Pôle «Travail » et «Concurrence, consommation, répression des fraudes, métrologie », 3 avril 2013).

On peut ici supposer que le point de vue des acteurs interrogés et leur adhésion au dispositif mis en place varient selon leur degré d'intervention et leur position dans le schéma de la gouvernance «emploi-formation » mis en place initialement. Ce dernier point renforce l'idée de la nécessité d'une concertation la plus large possible et d'une forte anticipation avec les acteurs concernés dès le début de la mise en œuvre des chantiers de grands projets d'infrastructure.

\section{CONCLUSION}

Plusieurs enseignements peuvent être tirés de la phase « recrutements » du système «emploiformation ». Tout d'abord, le dispositif mis place fait émerger des jeux d'échelle entre les acteurs. L'analyse de sa gouvernance a mis en évidence la prégnance des acteurs nationaux dans l'impulsion des décisions et des objectifs à atteindre. Les acteurs locaux ont été sollicités dans un second temps pour favoriser la mise en œuvre de ces décisions. Cela s'est traduit par la signature d'accords-cadres à l'échelle régionale et départementale ayant pour objectif de définir les missions de chaque acteur.

Plus largement, le processus de construction de la coordination fait apparaître quatre étapes successives et parfois concomitantes ayant abouti à la gouvernance publique/privée que nous venons de décrire. Tout d'abord, le concédant a posé formellement le cadre d'un environnement partenarial autour des questions d'emploi et de formation relatives au projet LGV SEA. Ces règles du jeu ont comme particularités d'impliquer d'un côté des partenaires géographiquement proches et de l'autre un concédant national, ancré localement par l'existence du projet. La proximité institutionnelle ainsi établie, à l'écart du constructeur, a nécessité pour ce dernier d'activer une stratégie de création de confiance avec les acteurs locaux pour aboutir à son objectif 
de recrutements, en s'appuyant sur les relations sociales interpersonnelles et interorganisationnelles. Cette étape essentielle et clairement explicitée par le constructeur a débouché sur l'élaboration d'un cadre institutionnel plus détaillé portant sur les missions de chaque partenaire. A partir de cette proximité institutionnelle et de la relation de confiance animant les partenaires, la dernière étape d'élaboration du processus de gouvernance a consisté en la définition d'une organisation commune et acceptée par tous, marquant l'apparition d'une proximité organisationnelle.

Le dernier enseignement qui ressort de cette analyse tient au rôle central du constructeur dans le pilotage de la politique «emploi-formation ». Grâce à l'appui de l'État, et après avoir construit une véritable démarche partenariale avec les acteurs locaux, celui-ci a pu répondre au mieux aux intérêts qui étaient les siens. Au regard de l'opinion des différents partenaires, et bien que n'ayant pas entièrement satisfait les intérêts de toutes les parties prenantes, on peut considérer que la coordination «emploi-formation » mise en œuvre dans la phase de recrutement a plutôt bien fonctionné pour remplir le principal objectif qui lui était assigné : répondre aux besoins de maind'œuvre qualifiée du constructeur. Le second objectif de cette coordination, qui est chronologiquement postérieur au premier, pose la question de la sécurisation des parcours professionnels des ressources humaines produites conjointement une fois leur mobilisation sur la construction terminée. En termes d'image, cette phase représente un enjeu considérable pour le constructeur. Pour l'État et le Conseil Régional, elle est politiquement sensible puisque questionnant le financement public d'une ressource locale utilisée par un groupement privé. Elle questionne la capacité des acteurs du territoire à remobiliser les ressources créées dans une logique de développement économique à moyen terme.

Pour répondre à cet objectif, les acteurs se sont appuyés sur la confiance et les proximités créées lors de la phase de recrutement. L'accord-cadre du 1/07/2011 a ainsi été renouvelé dans le cadre d'une plateforme d'appui aux mutations économiques spécifiquement dédiée au projet LGV SEA par signature d'un accord cadre le 4 décembre 2013. Cette plateforme « constitue (...) un outil d'anticipation de l'après LGV SEA » (Accord cadre relatif à la mise en place d'une plateforme territoriale d'appui aux mutations économiques LGV SEA, 2013). Le renouvellement de cet accord se traduit par un élargissement du nombre de parties prenantes (24 au total) autour des quatre principaux partenaires de la phase de recrutement: Pôle Emploi, COSEA, l'État, le Conseil Régional de Poitou-Charentes. Ainsi, d'une concentration du système de gouvernance pendant la phase de recrutement autour du duo COSEA/Pôle Emploi, on observe une déconcentration dans la phase de redéploiement, matérialisée par un partage des responsabilités plus marqué. Cette nouvelle organisation institutionnelle a d'ailleurs interpelé certaines organisations syndicales qui y voyaient un désengagement du constructeur (CGT PoitouCharentes, 2014). Par ailleurs, la gouvernance du dispositif pour la phase de redéploiement renforce le poids du niveau global par rapport à l'échelle locale. En effet, l'accord-cadre signé doit répondre aux exigences du Pacte national pour la Croissance, la compétitivité et l'emploi. 
Dans ce contexte, et en rapport avec les objectifs qui étaient assignés à la coordination «emploiformation » dans la phase « reclassements », l'enjeu principal pour évaluer la qualité du système de gouvernance réside dorénavant dans l'analyse du devenir professionnel des personnels locaux du projet SEA. Au printemps 2015, Pôle Emploi Poitou-Charentes indiquait que 49\% des 734 exsalariés de COSEA ou de ses sous-traitants accompagnés par leurs soins avaient retrouvé un emploi (CDI, CDIC ou période d'essai). «1 300 autres sont sortis du dispositif et ont sans doute retrouvé un emploi » (Courrier de l'ouest, 2015). Afin d'affiner cette analyse et de se prononcer sur l'efficacité de cette coordination pour la phase de redéploiement, une enquête sur la trajectoire professionnelle d'anciens salariés locaux du projet SEA a été lancée et fera l'objet d'un prochain article.

\section{REFERENCES}

Berion, P. (2002). La construction d'une grande infrastructure de transport et ses premiers effets territoriaux : le cas de l'autoroute A39, section de Dole à Bourg-en-Bresse. Géocarrefour, 77(1), 7-20.

Courrier de l'ouest (2015). La Ligne à grande vitesse a fait reculer le chômage ! Consulté le 21 avril 2015, à l'adresse http://www.courrierdelouest.fr/actualite/deux-sevres-la-lgv-a-fait-reculer-le-chomage-20-042015-216371

Coux, P.-D. (2012). Concession de la LGV Sud Europe Atlantique Tours-Bordeaux : un succès qui en appelle d'autres. Infrastructures et mobilité.

CGT Poitou-Charentes. (2014). Dispositif de reconversion des salariés du chantier LGV : la Cgt gagne sa participation. Consulté le 21/04/2015, à https://cgtpoitoucharentes.wordpress.com/2014/01/31/dispositif-de-reconversion-des-salaries-du-chantierlgv-la-cgt-gagne-sa-participation/

Dupuy, C., \& Torre, A. (2004). Confiance et proximité. In Economie de proximités, 65-87.

Dupuy C., Leroux I., Wallet F., 2003, Conflits, négociation et gouvernance territoriale, Droit et Société, n54, 50-63.

État, Pole Emploi, COSEA, Conseil régional de Poitou-Charentes. (2011). Convention de partenariat : retombées économiques et sociales du chantier.

État, Pole Emploi, COSEA, Conseil régional de Poitou-Charentes. (2013). Accord cadre relatif à la mise en place d'une plateforme territoriale d'appui aux mutations économiques LGV SEA.

Eiffage (2015). La ligne à grande vitesse Bretagne Pays de La Loire. Consulté le 22 avril 2015, à l'adresse http://www.ere-lgv-bpl.com/home

Ferrary, M. (2010). Dynamique des réseaux sociaux et stratégies d'encastrement social. Revue d'économie industrielle, (129-130), 171-202.

Fouqueray, E. (2016). Impact économique de la construction de la LGV SEA Tours-Bordeaux sur les régions traversées. Revue d'économie régionale et urbaine, $\mathrm{N}^{\circ} 2$, 385-416

Fouqueray, E. (2016). Evaluation de l'impact économique de court terme et de moyen terme des chantiers de grandes infrastructures de transport : Le cas de la LGV SEA Tours-Bordeaux. Thèse de doctorat, Université de Poitiers. 
Gilly, J.-P., Leroux, I., \& Wallet, F. (2004). Gouvernance et proximité. In Economie de proximités, 187-206.

Gilly, J.-P., \& Perrat, J. (2003). La dynamique institutionnelle des territoires : entre gouvernance locale et régulation globale. Cahiers du GRES.

Granovetter, M. (1985). Economic Action and Social Structure: The Problem of Embeddedness. American Journal of Sociology, 91(3), 481-510.

Grossetti, M. (2004). Sociologie de l'imprévisible. Dynamiques de l'activité et des formes sociales.

Hasiak, S., Jouve, N., \& Boijard-Lafont, B. (2007). Territoires et Seine-Nord Europe. Retour d'expériences autour de l'organisation, de la gestion et des retombées économiques de grands chantiers d'infrastructures de transport. CETE Nord Picardie.

Lempereur, A., \& Fouqueray, E. (2014). Les effets induits de la construction de la LGV SEA pour les départements traversés: une analyse intermédiaire des modes de vie et de consommation des compagnons. Observatoire socio-économique de la LGV SEA - LISEA.

Manceau, E., 2011, La politique régionale européenne et l'aménagement du territoire en Italie. L'exemple de la région Campanie. Thèse de doctorat, Université Paris-Diderot.

Mortensen, D. T., \& Pissarides, C. A. (1994). Job Creation and Job Destruction in the Theory of Unemployment. The Review of Economic Studies, 61(3), 397-415.

Organisation de coopération et de développement économiques. (2002). Investissements en infrastructure de transport et développement régional. Paris: OCDE.

Pecqueur, B., \& Zimmermann, J.-B. (2004). Economie de proximités. Paris: Lavoisier : Hermes Science. Perrat, J., \& Zimmermann, J.-B. (2003). Stratégies des firmes et dynamiques territoriales. Etudes de la documentation française.

Pôle Emploi (2015), Nos missions - Pôle emploi. Consulté 20 avril 2015, à l'adresse http://www.poleemploi.org/poleemploi/nos-missions-@/13840/view-category-13840.html

Quintin, P. (2007). Droit du travail, droit social et grands chantiers de construction : l'expérience française du tunnel sous la manche (1987 - 1996).

Raveyre, M. (2005a). Mondialisation et ancrage territorial: les nouvelles stratégies des grandes entreprises. Laboratoire d'Études Sociologiques des Transformations et Acculturations des Milieux Populaires.

Raveyre, M. (2005b). Restructurations, grands groupes et territoires. Géographie, économie, société, 7(4), 333-346.

Réseau Ferré de France. (2010). Les retombées économiques et sociales du chantier. 